\title{
Identification of Methylation Profiles of Cancer-related Genes in Circulating Tumor Cells Population
}

\author{
ANNA LYBEROPOULOU ${ }^{1}$, MICHAIL GALANOPOULOS ${ }^{2}$, GERASIMOS ARAVANTINOS ${ }^{3}$, \\ GEORGE E. THEODOROPOULOS ${ }^{4}$, EVANGELOS MARINOS ${ }^{1}$, \\ EFSTATHIOS P. EFSTATHOPOULOS ${ }^{5}$ and MARIA GAZOULI ${ }^{1,5}$ \\ ${ }^{1}$ Department of Basic Medical Sciences, Laboratory of Biology, School of Medicine, \\ National and Kapodistrian University of Athens, Athens, Greece; \\ ${ }^{2}$ Department of Gastroenterology General Hospital of Athens "Evaggelismos" Athens, Greece; \\ ${ }^{3}$ Department of Medical Oncology, "Agii Anargiri" Cancer Hospital, Athens, Greece; \\ ${ }^{4}$ First Propaedeutic Surgical Department, Medical School, \\ National and Kapodistrian University of Athens, Athens, Greece; \\ ${ }^{5}$ Department of Radiology, School of Medicine, National and Kapodistrian University of Athens, Athens, Greece
}

\begin{abstract}
Background: We performed an epigenetic analysis of the first exon of the hVIM gene and the SFRP2 in circulating tumor cells (CTCS) and correlation with the corresponding primary colorectal cancer $(C R C)$ tissue. Patients and Methods: CTCs detection in 52 colorectal cancer patients was managed by a multi-marker immunomagnetic method with the use of quantum dots $(Q D s)$. To determine methylation levels we used highresolution melting (HRM) technology. Results: In the case of VIM we found $76.9 \%$ methylated samples, compared to $53.8 \%$ in tissue samples. Regarding SFRP2 promoter methylation levels in tissue and CTCs samples, $67.3 \%$ and $73.1 \%$, were found methylated respectively. Correlation analysis of methylation levels with KRAS and BRAF mutations (performed in our previous study) demonstrates that high-methylation epigenotype strongly correlates to BRAF mutation. Conclusion: CTCs is a promising diagnostic tool. The combination of genetic mutations and epigenetic aberrations specifically in CTCs, will ameliorate CRC diagnosis in the future.
\end{abstract}

CTCs are a population of rare cells disseminated from the primary tumor that travel the bloodstream in order to form micrometastases in distant tissues. Therefore, it is evident

Correspondence to: Maria Gazouli, Ph.D., Associate Professor of Molecular Biology, School of Medicine, National and Kapodistrian University of Athens, Michalakopoulou 176, 11527 Athens, Greece. Tel/Fax: +302107462231, e-mail: mgazouli@med.uoa.gr

Key Words: CTCs, colorectal cancer, CTCs isolation, quantum dots, molecular characterization, methylation profile. that the detection of CTCs, in a blood sample of a cancer patient, may serve as an alternative to the biopsy. Liquid biopsies seem to be a very promising tool to be used as quick, non-invasive tests for the management of cancer. Several studies the past decade have demonstrated that the detection of CTCs in the blood indicates the initiation of the metastatic process or the relapse of the disease after treatment. CTCs enumeration is already offering in clinical practice prognostic and predictive information regarding cancer progression, treatment selection and patient stratification (1). However, it is shown that CTCs population is a heterogeneous population and only a subpopulation of CTCs can survive, reach distant organs and form preangiogenic micrometastasis. To date, it is hypothesized that CTCs are comprised of three main subpopulations; CTCs that are epithelial-derived, those that hold EMT (Epithelial to Mesenchymal Transition) features and finally the stemcell like CTCs with each subpopulation expressesing different biomarkers (2).

Consequently, there is no general consensus about the most accurate method to use for the detection and isolation of these rare cells, mainly because of the lack of a specific marker for their identification. Therefore, it is now more evident that the biomolecular characterization of CTCs could prove to be an effective tool, possibly in combination with counting of CTC numbers, for personalized treatment and clinical surveillance, patient stratification and monitoring of disease status. Finally, CTCs characterization aims to answer critical questions about the biology of CTCs, of tumor biology and aggressiveness and identification of therapeutic targets $(3,4)$.

Molecular characterization is based on the identification of cancer-specific biomarker expression in CTCs, their 
mutational analysis and their epigenetic analysis, which is still a novel area of research in CTCs. Recently our group defined the mutational analysis of specific cancer-related SNPs in CTCs, using a multi-marker immunomagnetic approach with the use of QDs (Quantum Dots) in order to isolate a pure CTCs population that will contain all three subpopulations of CTCs $(5,6)$. QDs have the ability to emit different colors in narrow bands in a size-dependant way, a fact that makes them an excellent tool for multiplex imaging. In this study we aimed to use the same immunomagnetic method, in order to identify for the first time the methylation profile of colorectal cancer (CRC) cancer-specific genes, Vimentin (VIM) and Secreted frizzled-related protein 2 (SFRP2) in CTCs DNA and compared to the corresponding tissue sample. VIM and SFRP2 methylation is associated to CRC development and is suggested as specific CRC biomarker. Although methylation profile of CTCs is a promising tool, epigenetic CTCs studies are still very limited to be able to develop novel epigenetic biomarkers for cancer management and further studies are needed.

\section{Patients and Methods}

Patients. Peripheral blood samples and tumor tissue biopsies were obtained from 52 metastatic CRC patients before initiation of any treatment, who gave informed consent to be included in this study. Additionally, 9 healthy samples were used as control group. Written informed consent was obtained from each patient or their families concerning the samples involved in the study. The research was approved by the Ethics committee of Atticon University Hospital, School of Medicine, University of Athens, Greece under the general title "Molecular Characterization of Circulating Tumor Cells" (1586/27/1/14). Peripheral venous blood was sampled immediately after patients were anaesthetized and prior to the commencement of surgery. In all patients, an intravenous cannula was used to collect blood into 7-ml vacutainers containing sodium ethylenediaminetetraacetic acid (EDTA), discarding the first 7-ml aliquot of blood to reduce the risk of contamination of blood by skin epithelial cells. Three 30-ml samples were then collected at one-minute intervals. Human peripheral blood monocellular cells (PBMCs) were isolated from peripheral blood using Ficoll-Hypaque PLUS reagents (Amersham Bioscienses, Little Chalfont, NA, UK). Cells were counted manually using a Burker-Turk haemocytometer. Trypan blue $(0.4 \%$, Sigma, LO, UK) exclusion test was used to ensure cell viability was above $90 \%$ in experiments. All patients had detectable number of CTCs as verified by FACS ( $>2$ cancer cells). Primary tumor tissue samples were obtained during surgery, frozen immediately in liquid nitrogen and stored at $-80^{\circ} \mathrm{C}$ until DNA extraction (5).

Detection and enrichment of CTCs. The aim of the enrichment and the subsequent detection of CTCs is the isolation of a high purity CTCs population that will contain cancer cells from all CTCs subpopulations. For that reason, we used an immunomagnetic-based detection technique with the use of QDs (Qdot655, Qdot525, Qdot605) that contains two purification steps with the use of multiple biomarkers for the detection of epithelial-derived CTCs
(CK19, EpCAM), CTCs with EMT features (Vimentin, CD29) and the stem-cell like CTCs (CD133), as previously described $(5,6)$.

DNA isolation. DNA from the enriched CTC samples and from the corresponding tissue samples was isolated using the NucleoSpin Tissue kit (Macherey-Nigel, GmbH \& Co. KG, Düren, Germany) according to the manufacturer's instructions. In healthy samples DNA was isolated from blood, since no CTCs are detecte. The quality and quantity of extracted DNA was evaluated with NanoDrop 1000 Spectrophotometer (Thermo Fisher Scientific Inc).

Bisulfite conversion. Two micrograms aliquot of genomic DNA were converted using the EpiTect Bisulfite Kit (Qiagen, Germany) in a sample volume of $20 \mu \mathrm{RNase-free} \mathrm{water} \mathrm{and} \mathrm{a} \mathrm{total} \mathrm{volume}$ of $140 \mu \mathrm{l}$, together with the Bisulfite mix $(85 \mu \mathrm{l})$ and the DNA protect buffer $(35 \mu \mathrm{l})$. The bisulfite conversion thermal cycler conditions used are: denaturation for $5 \mathrm{~min}$ in $95^{\circ} \mathrm{C}$, incubation for $25 \mathrm{~min}$ in $60^{\circ} \mathrm{C}$, denaturation for $5 \mathrm{~min}$ in $95^{\circ} \mathrm{C}$, incubation for $85 \mathrm{~min}$ in $60^{\circ} \mathrm{C}$, denaturation for $5 \mathrm{~min}$ in $95^{\circ} \mathrm{C}$, incubation for 175 min in $60^{\circ} \mathrm{C}$, hold in $20^{\circ} \mathrm{C}$.

HRM Real-time PCR for DNA methylation status analysis. The methylation profile of colorectal cancer (CRC) cancer-specific genes, VIM and SFRP2 were analyzed using specific primers: F: GCGATGGTTTATTGTAAGTTGGTAGT, R: CTATCCGCCGA TTAAAAACTCCTA, annealing $\mathrm{T}_{\mathrm{a}} 54^{\circ} \mathrm{C}$, Product 127 bp and $\mathrm{F}$ : TTTCGGATTGGGTAAAATAAGT, R: CGCTACGCCCCCACA ATAT, Ta $60^{\circ} \mathrm{C}$, Product $158 \mathrm{bp}$, respectively for the two genes. We performed real-time PCR EpiTect HRM PCR Kit (Qiagen, Germany), high-resolution melting (HRM) technology for fast screening and accurate detection of changes in the $\mathrm{CpG}$ methylation status of bisulfite converted DNA using a Rotor Gene 6000 instrument (Corbett Research, Mortlake, Australia). Data were analyzed with Rotor gene 6000 series software 1.7 (Corbett Research, Mortlake, Australia). All samples were compared with the $100 \%$ bisulfite converted positive control DNA. The later was also used in a standard curve of serial dilutions (1:10 and 1:5 as internal dilution) in order to validate the DNA methylation status of the samples.

Statistical analysis. The comparisons were analyzed using $\chi^{2}$ test. All statistical analyses were carried out using GraphPad version 3.00 (GraphPad Software, San Diego, CA, USA). $p<0.05$ was considered significant.

\section{Results}

In this study, the methylation profile of specific regions of VIM and SFRP2 were analyzed for 52 colorectal cancer patients with detectable number of CTCs ( $>2$ cells), as validated by FACS. The CTCs samples contained epithelial origin cancer cells, stem-like cells and cells showing EMT features as demonstrated with QD-labeling (5). Detailed patient characteristics are depicted in Table I.

Methylation levels of SFRP2 gene in CTCs population DNA. Methylation of $\mathrm{CpG}$ islands in $S F R P 2$ promoter regions has been broadly used as marker for the noninvasive detection of $\mathrm{CRC}$, since methylation of the SFRP2 in cancer tissues and 
Table I. Histopathological characteristics of CRC patients.

\begin{tabular}{lc}
\hline Characteristic & Patients (N=52) \\
\hline Age (median, range) & $63(35-82)$ \\
Gender & 19 \\
$\quad$ Female & 33 \\
$\quad$ Male & \\
Primary tumor location & 11 \\
$\quad$ Rectum & 12 \\
Right colon & 29 \\
Left colon & \\
Differentiation & 6 \\
Well & 40 \\
Moderate & 6 \\
Poor & \\
TNM stage & 0 \\
I & 6 \\
II & 24 \\
III & 22 \\
IV &
\end{tabular}

fecal samples is positively associated with CRC. The 158-bp amplicon that is analyzed in this study is shown in Figure 1. The detection limit of methylation with Real-Time HRM PCR was determined in $2 \%$ methylation, which can be successfully detected in samples and controls (Figure 2). According to our results, SFRP2 promoter methylation levels in tissue and CTCs samples, $67.3 \%$ (35/52) with 15 high methylated samples and $73.1 \%(38 / 52)$ with 12 high methylated samples, was found methylated respectively. Healthy samples (9) are not methylated (1 tissue sample was found low methylated in SFRP2 promoter and none CTCs sample). At the same time, there is strong concordance $(90.3 \%$ with 3 samples nonmethylated in tissue and methylated in CTCs, 2 samples nonmethylated in CTCs and methylated in tissue) between the methylation status of the SFRP2 promoter region in CTCs and the corresponding tissue, suggesting that the analysis of methylation in CTCs of CRC patients is a very promising noninvasive alternative for $\mathrm{CRC}$ detection.

Methylation levels of VIM gene in CTCs population DNA. Methylation of $\mathrm{CpG}$ islands in VIM promoter or $\mathrm{CpG}$ on the first exon (exon 1) of the hVIM gene is detected in most cancer tissues. We chose to analyze the methylation levels in CpGs of the exon 1 (Figure 3) of $h$ VIM in CTCs DNA and the corresponding tissue, since hypermethylation of exon 1 is described in $60-80 \%$ cases of CRC tumors, absent in normal colon epithelium and more specific CRC methylation biomarker compared to VIM promoter methylation. The detection limit of methylation with Real-Time HRM PCR was determined in $2 \%$ methylation, which can be successfully detected in samples and controls (Figure 4). According to our results, the methylation level of exon 1 hVIM in CRC tissue and CTCs samples was found $53.8 \%(28 / 52)$ with 2 samples found highly methylated (20-50\% methylation) and $76.9 \%$ (40/52) with 10 high methylated samples, respectively. In addition, it was found a $69.2 \%$ concordance between methylation level of CTCs and tissue (1 sample methylated in tissue and not in CTCs, 15 methylated in CTCs and not in tissue), suggesting a possible non-invasive liquid biopsy alternative for $\mathrm{CRC}$. On the other hand, 30.8\% discordance can be explained by the fact that CTCs gain various genetic alterations while in bloodstream, so that their phenotype does not correlate nor to that of the primary tumor, neither to the metastatic one. Thus, our methodology detected with $76.9 \%$ sensitivity hVIM methylation in the CTCs of cancer patients compared to healthy controls (1 sample was found low methylated, $2 \%$ ). So, $h$ VIM region in CTCs is demonstrated to be detected in $76.9 \%$ of patients with 10 high methylated samples compared to tissue samples $(53.8 \%$ with 2 high methylated samples).

Correlation of methylation levels and genetic mutations. In a previous study (5), we examined the genetic mutation status of CTCs in CRC in the same set of patients used in the present study. Mutations were identified for RAS, BRAF, CD133 and plastin as described in our previous study (5). Therefore, in this study we tried to compare the methylation levels of each sample with the corresponding mutational status for cancer associated mutations in $R A S$ and $B R A F$, since $\mathrm{CIMP}^{+} \mathrm{CRC}$ demonstrates strong association with these mutations $(5,7)$. No statistical significant correlation was found between methylation levels and CD133 and plastin mutations. Positive methylation of $S F R P 2$ promoter site seems to be significantly correlated with the existence of a $R A S$ mutation in codon 12 in tissue samples $(p<0.001)$, as well as in CTCs samples $(p<0.001)$. No significant correlation was found for exon 1 VIM methylation. Although no correlation was found between positive methylation of SFRP2 promoter site or exon 1 VIM and $B R A F$ V600E mutation, hypermethylation (methylation $>20 \%$ ) of both genes was found significantly correlated with $B R A F$ mutation $(p<0.001$ and $p<0.01$ for $S F R P 2$ in tissue an CTCs respectively, $p<0.05$ and $p<0.01$ for VIM in tissue an CTCs respectively). Our results also revealed that in CTCs, 30 out of 52 samples are co-methylated (co-methylation in SFRP2 promoter and VIM exon 1 ), while 22 out of 30 co-methylated samples hold $R A S$ mutation. In tissue samples on the other hand only 21 out of 52 are co-methylated and 14 hold RAS mutation, intensifying the importance of CTCs in cancer diagnosis.

\section{Discussion}

CTCs are a population of cells that escape from the primary tumor and travel the bloodstream to form micrometastases in distant tissues. Liquid biopsies seem to be a very promising tool in order to be used as quick, non-invasive tests for the 

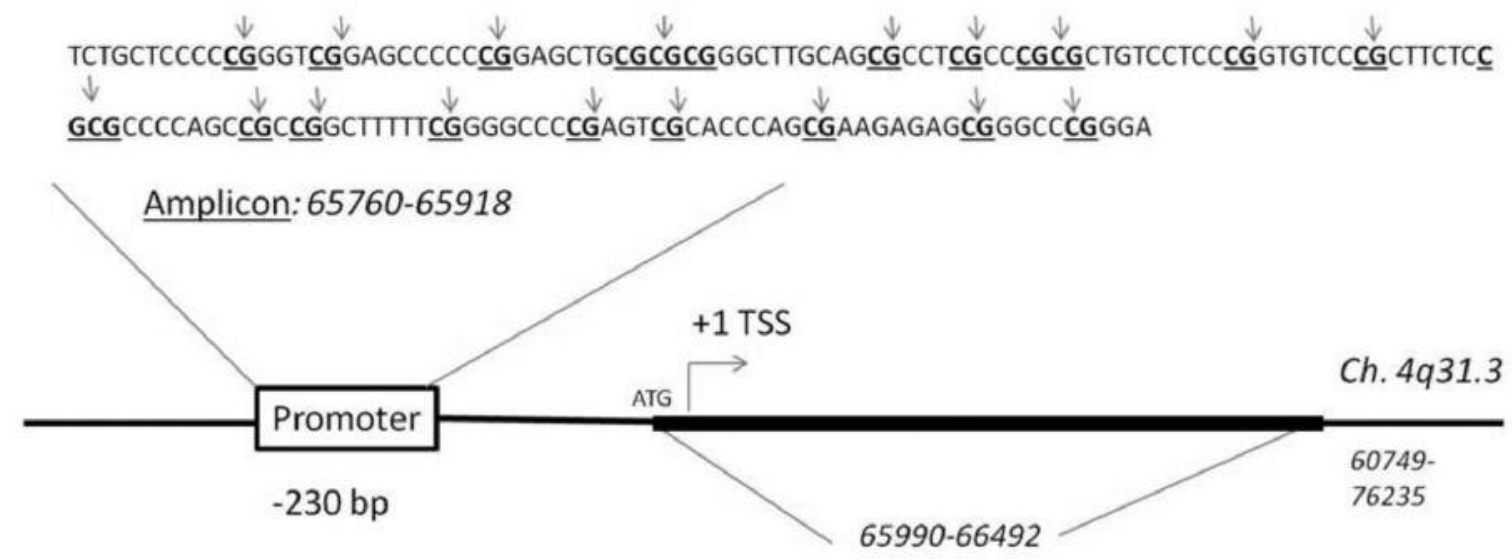

Figure 1. Position of CpGs of the 158 bp amplicon in the promoter of SFRP2 gene. The sequence was obtained from NCBI NG_046941.1 with the SFRP2 gene spanning 60749-76235 bp and the amplicon spanning 65760-65918 bp. The promoter region was identified in relation to the ATG translational start site (TSS), using the Genomatix MatInspector analysis.

management of cancer. Herein, we showed for the first time that SFRP2 and VIM are methylated in CTCs of colorectal cancer patients. Thus, we demonstrated that important tumor suppressor genes are methylated and probably downregulated not only in tissue samples, but even more in CTCs. DNA methylation has many advantages as cancer biomarker because of the stability in the detection and the early detection of cancer events in a sample. Furthermore, CTCs are proven to be a reliable and easily accessible sample that can be used in cancer management, especially compared to currently used invasive diagnostic tools in colorectal cancer management. In this study, CTCs detection is managed by the use of a multi-marker immunomagnetic method with the use of QDs. This method has already been standardized in our laboratory (6) and recently used for the mutational analysis of cancer-specific genes of CTCs (5). The method offers a CTCs population of high purity that contains all subpopulations of interest in CTCs. In this study the methylation profile of $\mathrm{CpG}$ islands on the first exon (exon 1) of the hVIM gene and $\mathrm{CpG}$ islands in SFRP2 promoter regions are analyzed and subsequently correlated with the corresponding primary CRC tissue sample. Methylation is a procedure that can be carried out either in the promoter sites of a gene or in the main body of a gene. $\mathrm{CpG}$ islands are sites in the promoters that when methylated, the corresponding genes get silenced, while when unmethylated the gene is characterized as transcription active. On the other hand, into the body of the gene hypermethylated sites facilitate gene transcription at the correct start sites. Cancer cells take advantage of this function of the cell: many genes are hypermethylated in sites that transcription is not supposed to begin and hypomethylated in the right start sites; at the same time it is shown that promoters of proto- oncogenes are hypermethylated in order to get suppressed, while crucial genes in cancer progression are hypomethylated and thus inducted (8). Therefore, methylation aberrations can offer a promising cancer biomarker that will be able to monitor clonal progression during metastasis. More specifically, in CRC various genes are shown to be unmethylated and expressed in normal colon mucosa, while methylated and silenced in CRC.

SFRP2 is a crucial component of Wnt signaling cascade, the induction of which is an early event in CRC carcinogenesis. SFRP2 is a negative regulator of the pathway and is proven to be downregulated mostly because of the hypermethylation of its promoter (9). SFRP2 methylation has been studied in CRC tissue, feces and serum and is suggested to be a specific CRC biomarker (10). Regarding SFRP2 promoter methylation levels, we found a $67.3 \%$ $(35 / 52)$ with 15 high methylated samples and $73.1 \%(38 / 52)$ with 12 high methylated samples, methylated in tissue and CTCs samples respectively. Healthy samples (9) were not found methylated (1 tissue sample was found low methylated in SFRP2 promoter and none CTCs sample). At the same time, there is a strong concordance $(90.3 \%$ with 3 samples non-methylated in tissue and methylated in CTCs, 2 samples non-methylated in CTCs and methylated in tissue) between the methylation status of the SFRP2 promoter region in CTCs and the corresponding tissue. Our results propose the use of methylation analysis in CTCs of CRC patients as a very promising non-invasive alternative for CRC detection.

Methylation of $\mathrm{CpG}$ islands in VIM exon 1 is described in 60- $80 \%$ cases of CRC tumors, absent in normal colon epithelium and more specific CRC methylation biomarker compared to VIM promoter methylation. VIM is an intermediate filament protein associated to EMT (epithelial 
A

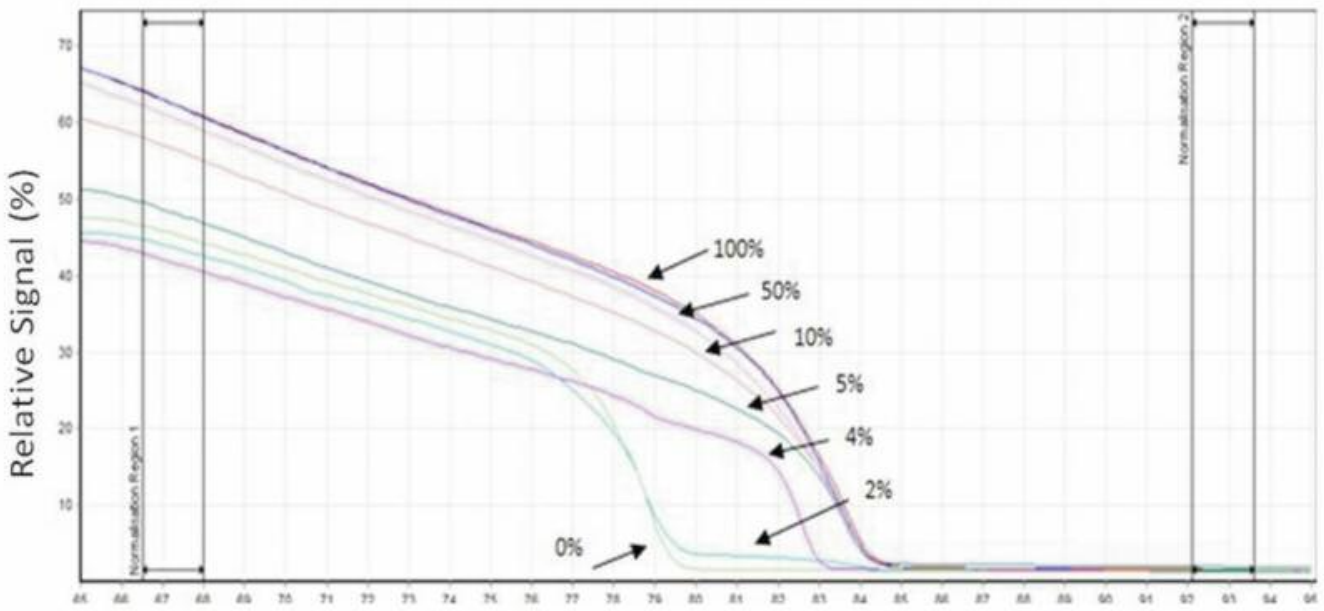

B

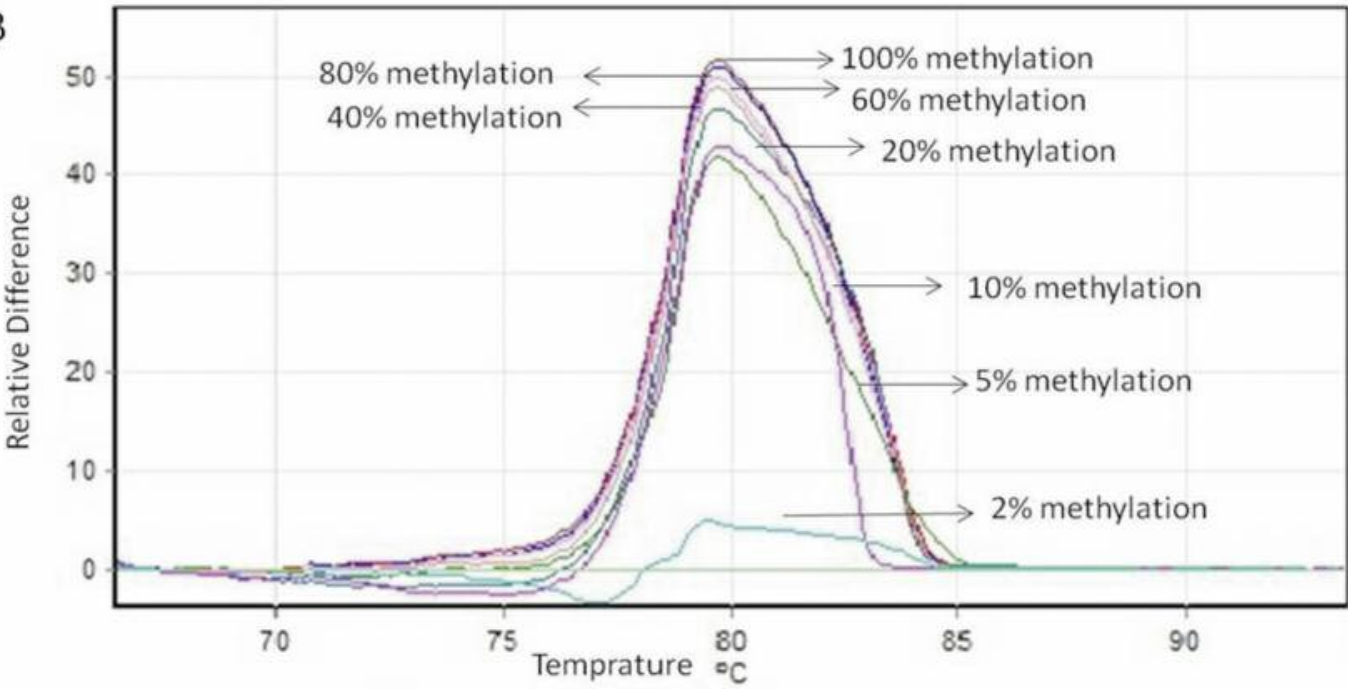

Figure 2. Representative plots of the HRM detection limit analysis for SFRP2. A) HRM fluorescence signal profiles of methylated DNA serial dilutions. B) Normalized temperature-shifted difference plots with fluorescence signals normalized in relation to 0\% methylated (unmethylated) control. Two percent methylation can be successfully detected.

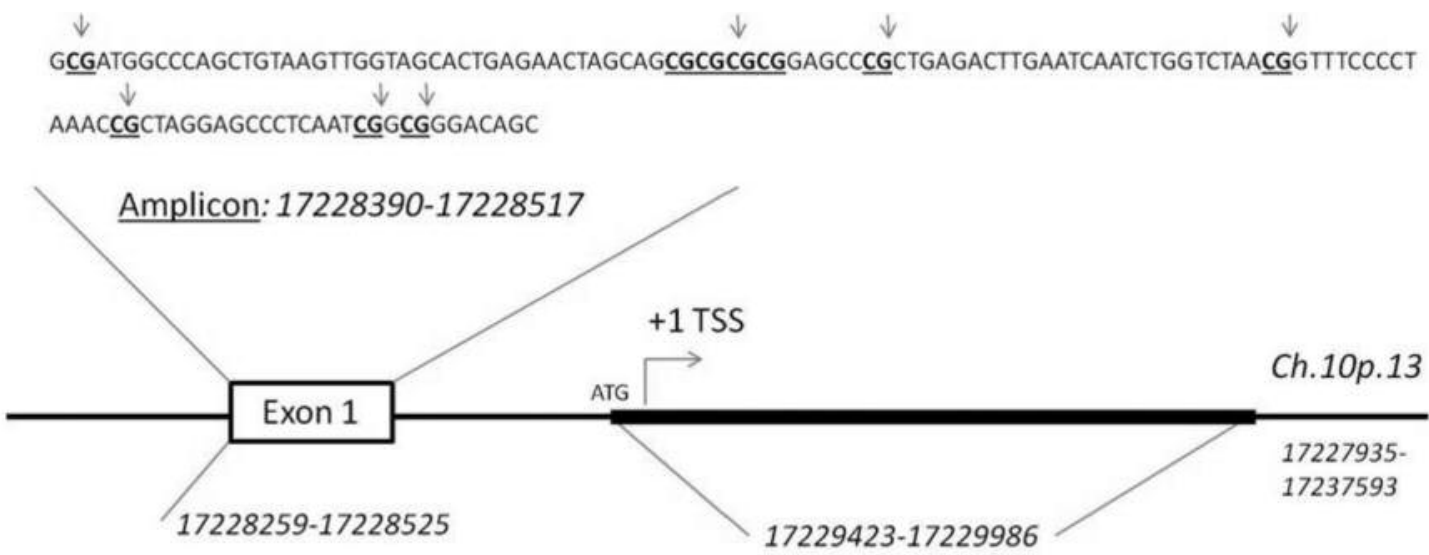

Figure 3. Position of CpGs of the 127 bp amplicon in exon 1 of VIM gene. The sequence was obtained from NCBI GRCh38.p7, NC_000010.11 with the VIM gene spanning 17227935-17237593 bp and the amplicon spanning 17228390-17228517 bp. 

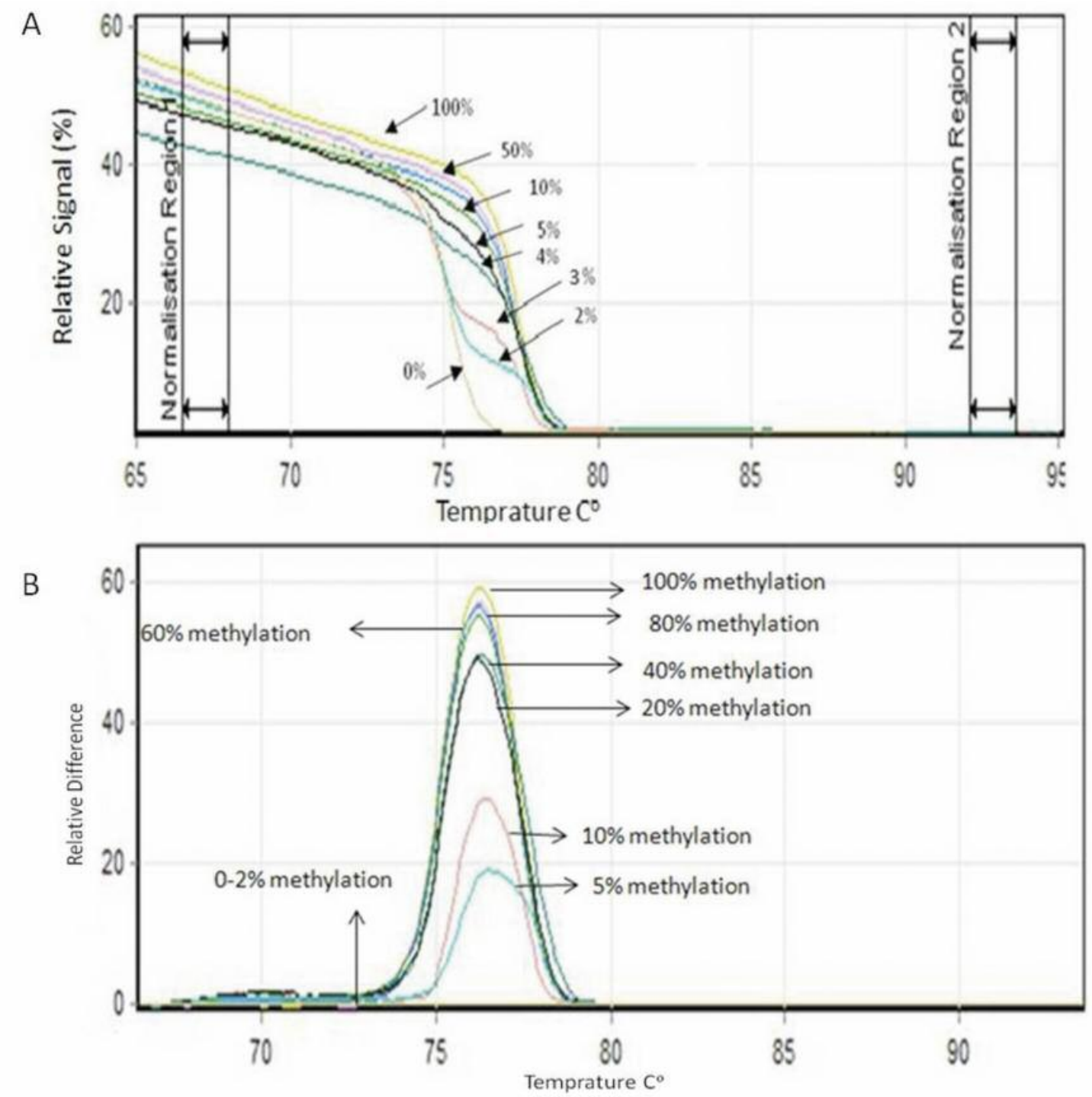

Figure 4. Representative plots of the HRM detection limit analysis for VIM. A) HRM fluorescence signal profiles of methylated DNA serial dilutions. B) Normalized temperature-shifted difference plots with fluorescence signals normalized in relation to $0 \%$ methylated (unmethylated) control. Two percent methylation can be successfully detected.

to mesenchymal transition), a crucial mechanism that participates in wound-healing cell responses and various pathological responses during cell invasion and cancer metastasis. Several studies have already demonstrated that VIM methylation leads to protein downregulation in many cancer types such as gastric, cervical, bladder, intestinal, hepatocellular, colorectal and that the methylation is absent in normal tissues (11-15). Furthermore, VIM methylation is suggested to be a useful cancer marker in serum and stool for the early detection of CRC $(16,17)$. Even though ColoSure $^{\mathrm{TM}}$ test (fecal VIM methylation assay) is already commercially available (18), VIM methylation tests in blood are still under investigation. Nevertheless, CTCs phenotype is considered to be a more representative diagnostic and prognostic tool in order to examine the "metastatic load" of the tumor, since this population includes cells deriving from the primary cancer and some of them will end up in metastatic tissues. According to our results, the methylation level of exon 1 hVIM in CRC tissue and CTCs samples was found $53.8 \%(28 / 52)$ with 2 samples found highly methylated (20-50\% methylation) and 76.9\% (40/52) with 10 high methylated samples, respectively. It is obvious, that methylation level of the gene in CTCs is aberrant compared to the methylation in tissue samples. Thus, the discordance 
(30.8\%) between CTCs and tissue methylation level can be explained by the fact that CTCs gain various genetic alterations while in bloodstream, so that their phenotype does not correlate nor to that of the primary tumor, neither to the metastatic one. However, probably CTCs phenotype can act alone as a prognostic and diagnostic tool that can be correlated with the existence or not of CRC or/and the therapeutic outcome, irrelevantly with the correlation of the phenotype with the primary tumor or the metastatic one. Therefore, our methodology detected with $76.9 \%$ sensitivity hVIM methylation in CTCs of CRC patients compared to healthy controls (1 sample was found low methylated, 2\%). In addition, in another study it is shown that unmethylaed VIM promoter in tissue samples is associated with improved prognosis in pancreatic cancer (19), while regarding CRC a Phase I/II study showed that higher levels of exon 1 VIM methylation in serum are associated with the obtainment of stable disease in CRC CIMP ( $\mathrm{CpG}$ islands promoter methylation phenotype)-high or positive $\left(\mathrm{CIMP}^{+}\right)$patients under chemotherapy. However, this study could not prove a potential prognostic or predictive role for the VIM epigenetic marker (20). CIMP phenotype in CRC is characterized by aberrant methylation in specific gene regions. CIMP phenotype, compared to $\mathrm{CIMP}^{-}$, is proven to be an independent prognostic marker of poor survival in rectal cancer patients, both for OS (overall survival) and DFS (disease-free survival) (21) and was associated with poorer survival also among CRC patients with MSI (Microsatellite Instability) $(8,22)$

Thus, we suggest that CTCs can be proved to be a more representative sample of the metastatic disease, since they are a source of metastatic cells and maybe their phenotype is associated with the CIMP phenotype. Therefore, we tried to compare the methylation levels of each sample with the corresponding mutational status for cancer associated mutations in $R A S$ and $B R A F$, examined recently in our lab in the same set of patients, since $\mathrm{CIMP}^{+} \mathrm{CRC}$ demonstrates strong association with these mutations $(5,7)$. It is currently believed that a combination of promising biomarkers can offer higher sensitivity regarding cancer management. The overall CTCs characterization can offer a panel of genes that hold various aberrations (epigenetic or mutational aberrations) that can act in combination as a CRC biomarker. Many studies, have also pointed-out the use of CTCs in combination with RAS mutations, in cancer monitoring during treatment, charachterizing CTCs as a dynamic source of tumor cells that can predict response to cancer therapy $(23,24)$. We believe that our study can offer important information towards this approach.

So far, molecular characterization of CTCs has mainly focused on their genomes, transcriptomes and proteomes, while epigenomic studies are relatively few. So in this study, we approached the methylation analysis of two CRC-specific genes, SFRP2 and VIM that are demonstrated to play a critical role in signaling pathways like growth and proliferation, invasiveness, EMT phenotype and stemness, in CTCs samples of CRC patients. We then compared the methylation status with that of the corresponding tissue. We found that CTCs could be a promising diagnostic tool, especially in the case of VIM with $76,9 \%$ methylated samples (10 high methylated samples included), compared to the $53,8 \%$ in tissue samples (2 samples found highly methylated). Our correlation analysis of metylation levels with $K R A S$ and $B R A F$ mutations demonstrates that highmethylation epigenotype strongly correlates to $B R A F$ mutation, while intermediate-methylation epigenotype correlates to KRAS mutation in the case of SFRP2 gene. Correlation of VIM hyper-methylation was found only for $B R A F$ mutation. Thus, we can hypothesize that $B R A F$ genetic mutation can somehow induce specific methylation especially in the case of VIM that the methylation in CTCs is higher or vice versa that gene methylation can "activate" existing genetic mutations. The latter can be the fact especially for SFRP2 promoter methylation that the concordance of methylations status between tissue and CTCs samples is high. The co-existance of genetic mutations, like KRAS and BRAF, with (hyper-) methylation of certain cancer-specific genes may lead to the development of tumorogenesis. Therefore, combination of genetic mutations and epigenetic aberrations specifically in CTCs, will be able to ameliorate CRC diagnosis in the future.

\section{References}

1 Barbazán J, Alonso-Alconada L, Muinelo-Romay L, Vieito M, Abalo A, Alonso-Nocelo M, Candamio S, Gallardo E, Fernández B, Abdulkader I, de Los Ángeles Casares M, Gómez-Tato A, López-López R and Abal M: Molecular characterization of circulating tumor cells in human metastatic colorectal cancer. PLoS One 7(7): e40476, 2012.

2 Barriere G, Fici P, Gallerani G, Fabbri F, Zoli W and Rigaud M: Circulating tumor cells and epithelial, mesenchymal and stemness markers: characterization of cell subpopulations. Transl Med 2(11): 109, 2014

3 Lianidou ES: Gene expression profiling and DNA methylation analyses of CTCs. Mol Oncol 10(3): 431-42, 2016.

4 Lowes LE and Allan AL: Recent advances in the molecular characterization of circulating tumor cells. Cancers 6(1): 595624, 2014.

5 Lyberopoulou A, Aravantinos G, Efstathopoulos EP, Nikiteas N, Bouziotis P, Isaakidou A, Papalois A, Marinos E and Gazouli M: Mutational analysis of circulating tumor cells from colorectal cancer patients and correlation with primary tumor tissue. PLoS One 10(4): e0123902, 2015.

6 Gazouli M, Lyberopoulou A, Pericleous P, Rizos S, Aravantinos G, Nikiteas N, Anagnou NP and Efstathopoulos EP: Development of a quantum-dot-labelled magnetic immunoassay method for circulating colorectal cancer cell detection. World J Gastroenterol 18(32): 4419-4426, 2012. 
7 Hinoue T, Weisenberger DJ, Lange CP, Shen H, Byun HM, Van Den Berg D, Malik S, Pan F, Noushmehr H, van Dijk CM, Tollenaar RA and Laird PW: Genome-scale analysis of aberrant DNA methylation in colorectal cancer. Genome Res 22(2): 271$282,2012$.

8 Jia M, Gao X, Zhang Y, Hoffmeister M and Brenner H: Different definitions of $\mathrm{CpG}$ island methylator phenotype and outcomes of colorectal cancer: a systematic review. Clin Epigenetics 8: 25, 2016.

9 Yang Q, Huang T, Ye G, Wang B and Zhang X: Methylation of SFRP2 gene as a promising noninvasive biomarker using feces in colorectal cancer diagnosis: a systematic meta-analysis. Sci Rep 6: 33339, 2016.

10 Tang D, Liu J, Wang DR, Yu HF, Li YK and Zhang JQ: Diagnostic and prognostic value of the methylation status of secreted frizzled-related protein 2 in colorectal cancer. Clin Invest Med 34: E88-E95, 2011.

11 Cong H, Yao RY, Sun ZQ, Qiu WS, Yao YS, Feng TT, Xin C, Liang $J$ and Yue LU: DNA hypermethylation of the vimentin gene inversely correlates with vimentin expression in intestinaland diffuse-type gastric cancer. Oncol Lett 11(1): 842-848, 2016.

12 Kisiel JB, Yab TC, Taylor WR, Chari ST, Petersen GM, Mahoney DW and Ahlquist DA: Stool DNA testing for the detection of pancreatic cancer: assessment of methylation marker candidates. Cancer 118(10): 2623-2631, 2012.

13 Kitamura Y, Shirahata A, Sakuraba K, Goto T, Mizukami H, Saito M, Ishibashi K, Kigawa G, Nemoto H, Sanada Y and Hibi $\mathrm{K}$ : Aberrant methylation of the Vimentin gene in hepatocellular carcinoma. Anticancer Res 31: 1289-1291, 2011.

14 Kitamura YH, Shirahata A, Sakata M, Goto T, Mizukami H, Saito M, Ishibashi K, Kigawa G, Nemoto H, Sanada Y and Hibi $\mathrm{K}$ : Frequent methylation of Vimentin in well-differentiated gastric carcinoma. Anticancer Res 29: 2227-2229, 2009.

15 Ulirsch J, Fan C, Knafl G, Wu MJ, Coleman B, Perou CM and Swift-Scanlan T: Vimentin DNA methylation predicts survival in breast cancer. Breast Cancer Res Treat 137(2): 383-96, 2013.

16 Shirahata A and Hibi K: Serum vimentin methylation as a potential marker for colorectal cancer. Anticancer Res 34(8): 4121-4125, 2014.

17 Lu H, Huang S, Zhang X, Wang D, Zhang X, Yuan X, Zhang Q and Huang Z: DNA methylation analysis of SFRP2, GATA4/5, NDRG4 and VIM for the detection of colorectal cancer in fecal DNA. Oncol Lett 8(4): 1751-1756, 2014.
18 Ned RM, Melillo S and Marrone M: Fecal DNA testing for Colorectal Cancer Screening: the ColoSure ${ }^{\mathrm{TM}}$ test. PLoS Curr 3: RRN1220, 2011.

19 Zhou YF, Xu W, Wang X, Sun JS, Xiang JJ, Li ZS and Zhang XF: Negative methylation status of vimentin predicts improved prognosis in pancreatic carcinoma. World J Gastroenterol 20(36): 13172-13177, 2014.

20 Overman MJ, Morris V, Moinova H, Manyam G, Ensor J, Lee MS, Eng C, Kee B, Fogelman D, Shroff RT, LaFramboise T, Mazard T, Feng T, Hamilton S, Broom B, Lutterbaugh J, Issa JP, Markowitz SD and Kopetz S: Phase I/II study of azacitidine and capecitabine/oxaliplatin (CAPOX) in refractory CIMP-high metastatic colorectal cancer: evaluation of circulating methylated vimentin. Oncotarget 7: 67495-67506, 2016.

21 Bae JM, Kim JH, Cho NY, Kim TY and Kang GH: Prognostic implication of the $\mathrm{CpG}$ island methylator phenotype in colorectal cancers depends on tumour location. Br J Cancer 109(4): 10041012, 2013.

22 Dahlin AM, Palmqvist R, Henriksson ML, Jacobsson M, Eklof V, Rutegard J, Obewrg A and Van Guelpen BR: The role of the $\mathrm{CpG}$ island methylator phenotype in colorectal cancer prognosis depends on microsatellite instability screening status. Clin Cancer Res 16(6): 1845-1855, 2010.

23 Musella V, Pietrantonio F, Di Buduo E, Iacovelli R, Martinetti A, Sottotetti E, Bossi I, Maggi C, Di Bartolomeo M, de Braud F, Daidone MG and Cappelletti V: Circulating tumor cells as a longitudinal biomarker in patients with advanced chemorefractory, RAS-BRAF wild-type colorectal cancer receiving cetuximab or panitumumab. Int J Cancer 137(6): 1467-1474, 2015.

24 Kalikaki A, Politaki H, Souglakos J, Apostolaki S, Papadimitraki E, Georgoulia N, Tzardi M, Mavroudis D, Georgoulias V and Voutsina A: KRAS genotypic changes of circulating tumor cells during treatment of patients with metastatic colorectal cancer. PLoS One 9(8): e104902, 2014. 